\title{
Reliability of Measuring Leg Segments and Joint Angles Using Smartphones during Aquatic Exercise
}

\author{
Dae Hee Lee ${ }^{1}$, Seulki Han ${ }^{2}$ \\ 'Department of Physical Therapy, U1 University, Yeongdong-gun, Chungbuk, Korea \\ ${ }^{2}$ Department of Physical Therapy, Daejeon Health Institute of Technology, Daejeon, Korea
}

Objectives: Aquatic therapy is a significant intervention method for both patients and healthy individuals. However, in clinical practice, quantitative measurements are rarely applied in aquatic therapy due to the disadvantages of submerging expensive instruments in water. In this study, we used readily available smartphones and armbands to measure leg segments and joint angles during aquatic gait and evaluated the reliability of these measurements. Methods: Waterproof smartphones were strapped to the trunk, thighs, and shanks of 19 healthy young adults using armbands. The angles of the trunk, thigh, and shank segments were measured during aquatic gait. The measurements were repeated 1 day later. The data were analyzed to obtain the angles of the hip and knee joints. Results: Measurement repeatability, calculated using the intraclass correlation coefficient (ICC), was the highest for the shank segment range of motion (ROM) (first $46.79^{\circ} \pm 5.50^{\circ}$, second $50.12^{\circ} \pm 9.98^{\circ}$, ICC $=0.78$ ). There was high agreement in trunk segment ROM (first $6.36^{\circ} \pm 1.42^{\circ}$, second $4.29^{\circ} \pm 1.83^{\circ}$, ICC $=0.73$ ), thigh segment ROM (first $33.49^{\circ} \pm 5.18^{\circ}$, second $37.31^{\circ} \pm 8.70^{\circ}$, ICC $=0.62$ ), and knee joint ROM (first $52.43^{\circ} \pm 11.26^{\circ}$, second $62.19^{\circ} \pm 16.65^{\circ}, \mathrm{ICC}=0.68$ ) and fair agreement in hip joint ROM (first $34.60^{\circ} \pm 4.71^{\circ}$, second $37.80^{\circ} \pm 7.84^{\circ}$, ICC $=0.59$ ). Conclusions: Smartphones can be used to reliably measure leg segments and joint angles during aquatic gait, providing a simpler method for obtaining these measurements and enabling the wider use of aquatic motion analysis in clinical practice and research.

Keywords: Smartphone, Articular Range of Motion, Gait, Knee Joint, Hip Joint

Submitted: February 24, 2021

Revised: 1st, April 26, 2021; 2nd, August 26, 2021

Accepted: September 24, 2021

\section{Corresponding Author}

Seulki Han

Department of Physical Therapy, Daejeon Health Institute of Technology, 21 Chungjeong-ro, Dong-gu, Daejeon 34504, Korea. Tel: +82-42-670-9338, E-mail: hsk@hit.ac.kr (https://orcid.org/00000002-2868-1165)

This is an Open Access article distributed under the terms of the Creative Commons Attribution Non-Commercial License (http://creativecommons.org/licenses/by$\mathrm{nc} / 4.0 /$ ) which permits unrestricted non-commercial use, distribution, and reproduction in any medium, provided the original work is properly cited.

(C) 2022 The Korean Society of Medical Informatics

\section{Introduction}

Aquatic therapy is recommended to maintain health or improve quality of life after injury [1]. Older patients who are unable to walk or who have difficulty walking due to balance and gait disturbances can walk in the water [2]. Aquatic gait training can improve or maintain gait ability $[3,4]$. The buoyancy of water counteracts gravity and compensates for the lack of muscle strength, reducing joint pain and stress and enabling partial weight-bearing gait training [5-8]. Furthermore, the viscosity of water decreases speed-dependent spasticity and enables the precise control of resistance through the speed of motion [3]. In addition, there is no risk of harmful falls in the water, allowing for more challenging tasks to be performed at earlier stages of recovery [9]. 
As walking is one of the most basic aquatic exercises [10], it is important to understand the dynamic changes that occur during aquatic gait to increase its therapeutic efficacy. A quantitative analysis can contribute to the improvement of a patient's gait ability [11]. Motion during aquatic gait can be analyzed on video, as several studies have reported [1215]. However, water turbulence, light refraction, air bubbles, and emulsion can occur even in favorable water conditions, decreasing the clarity of images [15-18]. Moreover, it is impractical to minimize waves and air bubbles by limiting pool use to one patient at a time, as pools are a limited resource. The use of expensive instruments underwater is also challenging, even after waterproofing [10], and not all cameras used in motion analysis systems are waterproof. For this reason, aquatic gait is usually evaluated visually and qualitatively by coaches or therapists, and quantitative data are not obtained [2,19]. Quantifying the characteristics of motion in water is challenging due to water interference, and the electronic devices used must be waterproof and readily available. These challenges must be overcome to measure the efficacy of aquatic exercises [20].

Health-related applications on smartphones have grown considerably in number and popularity [21] and are increasingly used in health management [22]. As smartphones are equipped with various sensors, including an accelerometer and a gyroscope sensor, they are appropriate tools for measuring the segment angle of the leg during walking [23]. Additionally, some smartphones are waterproof, and commercialized waterproofing instruments are readily available.

Previous studies have used smartphones to measure segments and joint angles during gait when walking on land. However, most studies measured segment angles in a static position or measured only one joint angle with the remaining trunk or segment completely fixed, and only a few studies obtained measurements in dynamic conditions [22]. Smartphones equipped with sensors for measuring movement are familiar to most users, making them a practical solution in terms of accessibility, convenience, portability, and affordability $[10,24]$. Smartphones can be used for measuring changes in the joint angles and the segments of the leg when walking underwater.

Recent studies have attempted to quantitatively assess aquatic gait. Mangia et al. [1] and Fantozzi et al. [2] measured segment angles during aquatic gait using magnetic sensors. However, no studies have measured joint and segment angles using a device that is easily accessible, such as a smartphone. This study aimed to investigate the reliability of leg segment and joint angle measurements obtained using smartphones during aquatic gait.

\section{Methods}

\section{Research Participants}

Based on the results of Furrer et al. [25], the number of participants was calculated using $G^{\star}$ Power software version 3.1.9.2 (Heinrich Heine Universität Düsseldorf, Germany) ( $a$ error probability of 0.05 , power of 0.90 ), which resulted in a total of 18 participants (actual power of 0.90 ). Subsequently, the final number of participants was increased to 19 individuals to account for dropout. The study was approved by the Daejeon Health Institute of Technology Institutional Review Board (No. 1041490-20200529-HR-002). The inclusion criteria for participants were as follows: (1) those who had not experienced falls in daily life due to ocular or vestibular diseases, (2) those who had no diseases or impairments of the musculoskeletal or nervous systems, (3) those who had no fear of water, and (4) those who voluntarily provided verbal and written consent for the present study.

\section{Research Facilities and Instruments}

The present study was conducted in a therapy pool of a rehabilitation hospital located in Daejeon, South Korea. The water depth was $110 \mathrm{~cm}$, and the pool area was $14 \mathrm{~m} \times 7 \mathrm{~m}$. The water temperature was $33^{\circ} \mathrm{C}$ and the atmospheric temperature was $24^{\circ} \mathrm{C}-27^{\circ} \mathrm{C}$ (Figure 1).

Segment angles were measured by strapping a Galaxy S8 (Samsung, Suwon, Korea) smartphone to the body and recording video. The smartphone had an Ingress Protection 68 (IP68) water-resistance rating, enabling it to withstand a water depth of $1.5 \mathrm{~m}$ for 30 minutes [26]. A tablet (MediaPad M3 Lite; Huawei, Shenzhen, China) was used to display the international standard time during videotaping. To prevent water damage caused by long periods of submergence, a commercially available waterproof smartphone case (applied phone model, Galaxy S8; Redpepper, China) with IP68 and ISO 9001 certification was used. Although the Galaxy S8 smartphone is waterproof, the case provided additional security. When inserting the smartphone into the case, a small piece of tissue was inserted alongside to prevent leakage into the case and to confirm upon that the inside of the case was kept waterproof throughout the experiment. Furthermore, while the smartphone fit closely in the waterproof case and was not prone to shaking, the addition of the tissue prevented further motion.

A 360 Season 3 Armband (Imcommerce Co., Seoul, Korea) was used to strap the smartphone to the participant. The 


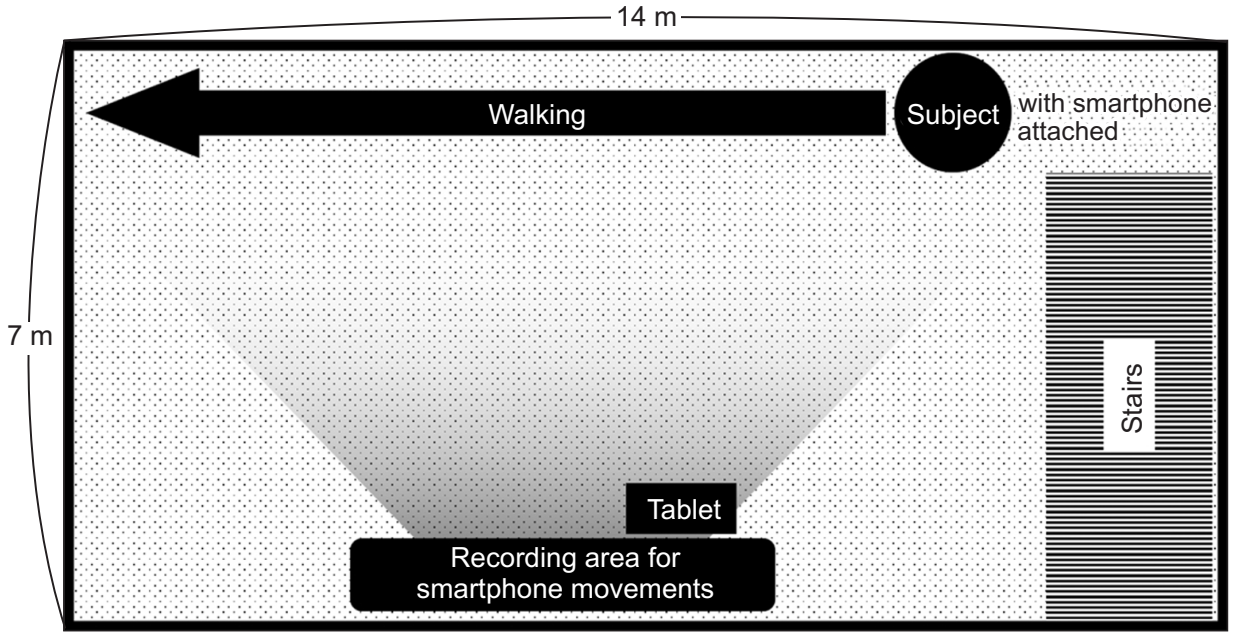

Figure 1. Pool layout and conditions. The water depth was $110 \mathrm{~cm}$, and the pool area was $14 \mathrm{~m} \times$ $7 \mathrm{~m}$. The water temperature was $33^{\circ} \mathrm{C}$ and the atmospheric temperature was $24^{\circ} \mathrm{C}$ $27^{\circ} \mathrm{C}$.

armband allows $360^{\circ}$ rotation of the smartphone, at $180^{\circ}$ either to the left or the right. The smartphone was attached along the coronal plane of the body, as the segment angles would be reversed at $0^{\circ}$ (vertical) if the smartphone was strapped along the body [24]. The armband was not long enough for some participants, and in these cases, Velcro was added to fully secure the smartphone around each body segment (Figure 2).

The Sensor Kinetics Pro application version 2.1.2 (Innoventions Inc., Houston, TX, USA) was used for the simultaneous extraction of acceleration and gyroscope values from the smartphone's sensors, conversion to rotation values, and recording of international standard time. Data from the smartphone were converted to .csv files and subsequently analyzed. The sampling rate was set to $50 \mathrm{~Hz}$, and the extracted values were synchronized using international standard time.

\section{Measurement Method}

Smartphones were strapped to the anterior aspect of the left thigh, the anterior aspect of the shank, and the posterior aspect of the trunk (waist) using armbands. Smartphones were placed in landscape orientation and aligned with the coronal plane so that the possible pitch value was $-90^{\circ}$. The participants slowly entered the therapy pool using the stairs and walked at a comfortable pace for approximately 3 minutes to adapt to the water. Thereafter, participants were instructed to walk at least seven gait cycles (14 steps) straight ahead in the therapy pool and to leave the pool via the stairs immediately after the measurements were obtained. The measurements were videotaped using a tablet that displayed the international standard time. Measurements were performed twice at 1-day intervals.
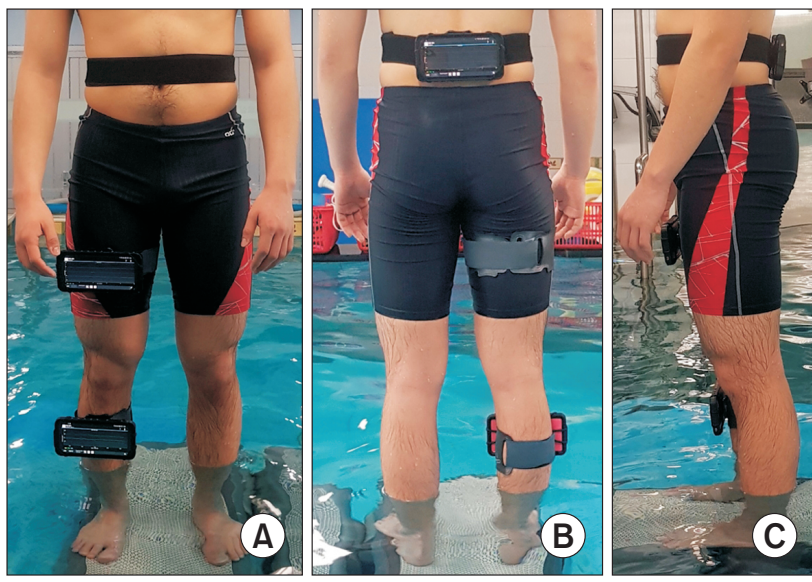

Figure 2. Trunk, thighs, and shanks strapped with smartphones: (A) front view, (B) back view, and (C) side view. Smartphones were strapped to the trunk, thighs, and shanks using armbands and Velcro.

\section{Statistical Analysis}

Excel (Microsoft, Redmond, WA, USA) and R software (v. 3.5.2, https://www.r-project.org/) programs were used to process and analyze the data. Statistical significance was set at $p<0.05$.

The time at which the left heel touched the bottom of the pool was considered the starting point of the gait cycle, and the time at which the left heel touched the bottom again was set as the end of the gait cycle. To calculate segment angles, the reference values measured in the standing position before the gait cycle were subtracted from the values measured at each time point. With the total gait cycle set at $100 \%$, data were extracted at points corresponding to every $5 \%$, resulting in 21 data points for each gait cycle. The hip joint angle was calculated by subtracting the trunk segment angle from the thigh segment angle, and the knee joint angle was calculated by subtracting the shank segment angle from the 
thigh segment angle. Differences between the maximum and minimum values for the trunk segment angle, thigh segment angle, shank segment angle, hip joint angle, and knee joint angle were used to calculate the range of motion (ROM) for each.

The intraclass correlation coefficient (ICC) [1,2] of the ROM for each item was calculated to examine the correlation between the first and second measurements. For ICC values, the following guidelines were used to interpret the results: values $>0.75$ represented excellent reliability; $0.60-0.75$, good reliability; $0.40-0.60$, fair reliability; and $<0.40$, poor reliability [27].

\section{Results}

This study included 19 participants (seven men, 12 women) with a mean age of $22.00 \pm 1.91$ years, a mean height of $167.26 \pm 8.97 \mathrm{~cm}$, and a mean weight of $66.53 \pm 14.65 \mathrm{~kg}$. Measurements of the trunk segment ROM (first $6.36^{\circ} \pm 1.42^{\circ}$, second $4.29^{\circ} \pm 1.83^{\circ}, \mathrm{ICC}=0.73$ ) and thigh segment ROM (first $33.49^{\circ} \pm 5.18^{\circ}$, second $37.31^{\circ} \pm 8.70^{\circ}$, ICC $=0.62$ ) obtained via the first and second measurements showed good reliability. Measurements of the shank segment ROM (first $46.79^{\circ} \pm 5.50^{\circ}$, second $50.12^{\circ} \pm 9.98^{\circ}$, ICC $\left.=0.78\right)$ showed excellent reliability (Table 1 ).

Measurements of knee joint ROM (first $52.43^{\circ} \pm 11.26^{\circ}$, second $62.19^{\circ} \pm 16.65^{\circ}$, ICC $=0.68$ ) obtained via the first and second measurements showed good reliability and fair

Table 1. Test-retest reliability of trunk, thigh, and shank segment ROM measurements

\begin{tabular}{lrrr}
\hline & \multicolumn{2}{c}{ ROM $\left(^{\circ}\right)$} & \multirow{2}{*}{ ICC } \\
\cline { 2 - 3 } & \multicolumn{1}{c}{ First } & \multicolumn{1}{c}{ Second } & \\
\hline Trunk & $6.36 \pm 1.42$ & $4.29 \pm 1.83$ & 0.73 \\
Thigh & $33.49 \pm 5.18$ & $37.31 \pm 8.70$ & 0.62 \\
Shank & $46.76 \pm 5.50$ & $50.12 \pm 9.89$ & 0.78 \\
\hline
\end{tabular}

Values are presented as mean \pm standard deviation.

ROM: range of motion, ICC: intraclass correlation coefficient.

Table 2. Test-retest reliability of hip and knee joint ROM measurements

\begin{tabular}{llll}
\hline & \multicolumn{2}{c}{$\left.\mathrm{ROM}^{\circ}{ }^{\circ}\right)$} & \multirow{2}{*}{ ICC } \\
\cline { 2 - 3 } & \multicolumn{1}{c}{ First } & \multicolumn{1}{c}{ Second } & \\
\hline Hip joint & $34.60 \pm 4.71$ & $37.80 \pm 7.84$ & 0.59 \\
Knee joint & $52.43 \pm 11.26$ & $62.19 \pm 16.65$ & 0.68 \\
\hline
\end{tabular}

Values are presented as mean \pm standard deviation.

ROM: range of motion, ICC: intraclass correlation coefficient. agreement with hip joint $\mathrm{ROM}$ (first $34.60^{\circ} \pm 4.71^{\circ}$, second $37.80^{\circ} \pm 7.84^{\circ}$, ICC $\left.=0.59\right)($ Table 2 , Figures 3,4$)$.

\section{Discussion}

This study investigated the reliability of leg segment and joint angle measurements obtained using smartphones during aquatic gait. We attached smartphones to the trunks, thighs, and shanks of 19 healthy young adults and measured the angles of these segments during aquatic gait to obtain the hip joint and knee joint angles. We found that smartphones are a reliable tool to measure the leg segments and

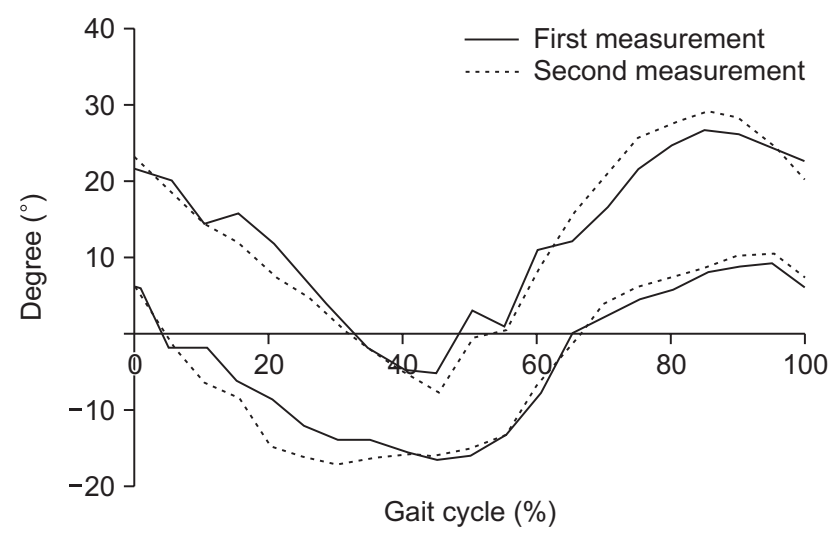

Figure 3. Hip joint ROM during aquatic gait. The two upper lines show the mean maximum values for all participants during the first (solid line) and second measurements (dotted line), and the two lower lines show the mean minimum values for all participants during the first (solid line) and second measurements (dotted line). ROM: range of motion.

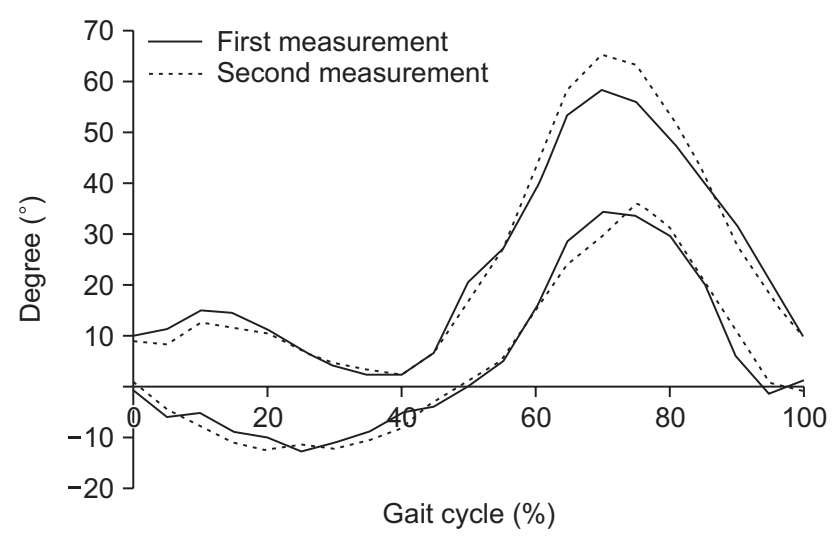

Figure 4. Knee joint ROM during aquatic gait. The two upper lines show the mean maximum values for all participants during the first (solid line) and second measurements (dotted line), whereas the two lower lines show the mean minimum values for all participants during the first (solid line) and second measurements (dotted line). ROM: range of motion. 
joint angles during aquatic gait. Therefore, this method can be used to obtain quantitative data in clinical practice and in research that requires aquatic motion analysis.

Previous studies have used accelerometers to develop practical and economical gait analysis devices. An accelerometer is small and can be controlled wirelessly through an additional device; therefore, it can be used for measurements in various settings $[28,29]$. However, accelerometers are generally unable to obtain joint angles. In contrast, smartphones are inexpensive, available to most individuals, and equipped with the sensors needed to measure segment angles. Free or inexpensive applications can be downloaded to smartphones to allow for the measurement of segment angles. Smartphones are thus more economically advantageous than other devices.

Jung et al. [4] reported a hip joint ROM of $19.65^{\circ}$ measured in water at the chest level, which differs significantly from our results. These conflicting results may be due to the differences in study methods, as the previous study used a treadmill with a rough surface and allowed participants to hold the rail during walking, whereas the present study allowed participants to walk comfortably on the bottom surface of the pool without auxiliary equipment. We found a higher value for hip joint ROM, which does not appear to be due to the buoyancy generated by the air present in the waterproof case, since the smartphone and waterproof phone case connected to the armband had negative buoyancy. Barela et al. [30] reported a knee joint ROM of $56.4^{\circ}$, measured in water at the xiphoid level, which is comparable to our results, using conditions similar to our study. Fantozzi et al. [2] measured joint angles using magnetic sensors during aquatic gait and obtained results similar to this study for hip joint ROM $\left(32.9^{\circ}\right)$ and knee joint ROM $\left(64.9^{\circ}\right)$.

This study has several limitations. Young and healthy participants were included, which may limit the generalizability of the results. We were unable to measure the movement of the ankle joint as we could not attach a smartphone to the foot. In future studies, patients of various ages and those with different conditions should be included, and technologies enabling communication between a separate sensor attached to the foot should be developed to study ankle joint motion. The water depth in this study was fixed at $110 \mathrm{~cm}$ as the therapy pool was rented after clinical hours. While some therapy pools are equipped with a system to control water depth, most of these facilities are not large enough for seven gait cycles and require expensive equipment, such as an underwater treadmill. We determined that this equipment was inappropriate for our study as the speed cannot be precisely controlled. The water reached between the waist and chest of all the participants in this study. Jung et al. [4] reported that a change in depth between the levels of the waist and chest had no significant effects on the spatiotemporal variables of gait. Unlike commercialized gait analysis instruments that are developed with their own software, gait analysis applications made for smartphones have not yet been commercialized [24]. Thus, the data had to be extracted and transferred to a computer for analysis. Future gait analysis applications for smartphones should develop a data analysis feature.

Another limitation of this study is that the validity of smartphone-based measurements in comparison to measurements taken using verified methods and equipment could not be determined. Further research should be conducted to confirm the validity of smartphone-based measurements for measuring joint angles during aquatic gait. Furthermore, methods should be developed to extract gait speed using information obtained through sensors that are present in smartphones, such as accelerometers.

Smartphones provide reliable measurements of leg segments and joint angles during aquatic gait and are an affordable option. While smartphones are not a substitute for existing and more expensive professional instruments, they can provide reliable and economic measurements of leg segments and joint angles during aquatic gait-as exemplified by the affordable and readily available method presented in this study.

\section{Conflict of Interest}

No potential conflict of interest relevant to this article was reported.

\section{Acknowledgments}

This work was supported by the Daejeon Health Institute of Technology (No. 2020003).

\section{ORCID}

Dae Hee Lee (https://orcid.org/0000-0001-9236-5776)

Seulki Han (https://orcid.org/0000-0002-2868-1165)

\section{References}

1. Mangia AL, Cortesi M, Fantozzi S, Giovanardi A, Borra D, Gatta G. The use of IMMUs in a water environment: instrument validation and application of 3D multi-body 
kinematic analysis in medicine and sport. Sensors (Basel) $2017 ; 17(4): 927$.

2. Fantozzi S, Giovanardi A, Borra D, Gatta G. Gait kinematic analysis in water using wearable inertial magnetic sensors. PLoS One 2015;10(9):e0138105.

3. Mercer JA, Applequist BC, Masumoto K. Muscle activity during running with different body-weight-support mechanisms: aquatic environment versus body-weightsupport treadmill. J Sport Rehabil 2014;23(4):300-6.

4. Jung T, Kim Y, Lim H, Vrongistinos K. The influence of water depth on kinematic and spatiotemporal gait parameters during aquatic treadmill walking. Sports Biomech 2019;18(3):297-307.

5. Dolbow DR, Farley RS, Kim JK, Caputo JL. Oxygen consumption, heart rate, rating of perceived exertion, and systolic blood pressure with water treadmill walking. J Aging Phys Act 2008;16(1):14-23.

6. Nakazawa K, Yano H, Miyashita M. Ground reaction forces during walking in water. In: Mutoh Y, Miyashita $\mathrm{M}$, Richardson $\mathrm{AB}$, editors. Medicine and science in aquatic sports. Basel, Switzerland: S. Karger AG; 1994. p. 28-34.

7. Murray JM, Hunter DL, Kelsey DD, Murray TD. Determination of the physiological effects of unloaded treadmill exercise. Cardiopulm Phys Ther J 1993;4(2):13-6.

8. Skelton DA, Dinan SM. Exercise for falls management: rationale for an exercise programme aimed at reducing postural instability. Physiother Theory Pract 1999;15(2):105-20.

9. Alikhajeh Y, Hosseini SR, Moghaddam A. Effects of hydrotherapy in static and dynamic balance among elderly men. Procedia Soc Behav Sci 2012;46:2220-4.

10. Silsupadol P, Teja K, Lugade V. Reliability and validity of a smartphone-based assessment of gait parameters across walking speed and smartphone locations: body, bag, belt, hand, and pocket. Gait Posture 2017;58:51622.

11. Masumoto K, Hamada A, Tomonaga HO, Kodama K, Amamoto Y, Nishizaki Y, et al. Physiological and perceptual responses to backward and forward treadmill walking in water. Gait Posture 2009;29(2):199-203.

12. Ceseracciu E, Sawacha Z, Fantozzi S, Cortesi M, Gatta G, Corazza S, et al. Markerless analysis of front crawl swimming. J Biomech 2011;44(12):2236-42.

13. Cortesi M, Fantozzi S, Gatta G. Effects of distance specialization on the backstroke swimming kinematics. J Sports Sci Med 2012;11(3):526-32.

14. McCabe CB, Sanders RH. Kinematic differences be- tween front crawl sprint and distance swimmers at a distance pace. J Sports Sci 2012;30(6):601-8.

15. Tamburella F, Scivoletto G, Cosentino E, Molinari M. Walking in water and on land after an incomplete spinal cord injury. Am J Phys Med Rehabil 2013;92(10 Suppl 2):e4-15.

16. Silvatti AP, Dias FA, Cerveri P, Barros RM. Comparison of different camera calibration approaches for underwater applications. J Biomech 2012;45(6):1112-6.

17. Silvatti AP, Cerveri P, Telles T, Dias FA, Baroni G, Barros RM. Quantitative underwater 3D motion analysis using submerged video cameras: accuracy analysis and trajectory reconstruction. Comput Methods Biomech Biomed Engin 2013;16(11):1240-8.

18. Magalhaes FA, Sawacha Z, Di Michele R, Cortesi M, Gatta G, Fantozzi S. Effectiveness of an automatic tracking software in underwater motion analysis. J Sports Sci Med 2013;12(4):660-7.

19. Mooney R, Corley G, Godfrey A, Osborough C, Newell J, Quinlan LR, et al. Analysis of swimming performance: perceptions and practices of US-based swimming coaches. J Sports Sci 2016;34(11):997-1005.

20. Masumoto K, Mercer JA. Biomechanics of human locomotion in water: an electomyographic analysis. Exerc Sport Sci Rev 2008;36(3):160-9.

21. Buechi R, Faes L, Bachmann LM, Thiel MA, Bodmer NS, Schmid MK, et al. Evidence assessing the diagnostic performance of medical smartphone apps: a systematic review and exploratory meta-analysis. BMJ Open 2017;7(12):e018280.

22. Longoni L, Brunati R, Sale P, Casale R, Ronconi G, Ferriero $G$. Smartphone applications validated for joint angle measurement: a systematic review. Int J Rehabil Res 2019;42(1):11-9.

23. Polechonski J, Nawrocka A, Wodarski P, Tomik R. Applicability of smartphone for dynamic postural stability evaluation. Biomed Res Int 2019;2019:9753898.

24. Lee D, Han S. Study on the reliability of smartphonebased measurements of leg segment and joint angles during gait. J Korean Soc Precis Eng 2019;36(3):293300.

25. Furrer M, Bichsel L, Niederer M, Baur H, Schmid S. Validation of a smartphone-based measurement tool for the quantification of level walking. Gait Posture 2015;42(3): 289-94.

26. Samsung Galaxy S [Internet]. Seoul, Korea: Samsung; c2021 [cited at 2022 Jan 4]. Available from: https://www. samsung.com/sec/smartphones/galaxy-s8/. 
27. Cicchetti DV. Guidelines, criteria, and rules of thumb for evaluating normed and standardized assessment instruments in psychology. Psychol Assess 1994:6(4):28490.

28. Lugade V, Fortune E, Morrow M, Kaufman K. Validity of using tri-axial accelerometers to measure human movement. Part I: posture and movement detection. Med Eng Phys 2014;36(2):169-76.
29. Fortune E, Lugade V, Morrow M, Kaufman K. Validity of using tri-axial accelerometers to measure human movement. Part II: step counts at a wide range of gait velocities. Med Eng Phys 2014;36(6):659-69.

30. Barela AM, Stolf SF, Duarte M. Biomechanical characteristics of adults walking in shallow water and on land. J Electromyogr Kinesiol 2006;16(3):250-6. 